\title{
Linear Integral Inequalities Involving Maxima of the Unknown Scalar Functions
}

\author{
By \\ Snezhana G. Hristova and Kremena V. Stefanova \\ (Plovdiv University, Bulgaria)
}

\begin{abstract}
This paper deals with some integral inequalities that involve the maximum of the unknown scalar function of one variable. The considered inequalities are generalizations of the classical integral inequality of Gronwall-Bellman. The importance of these integral inequalities comes from their wide applications in qualitative investigations of differential equations with "maxima" and is illustrated by some direct applications.

Key Words and Phrases. Integral inequalities, Maxima, Scalar functions of one variable, Differential equations with "maxima".

2000 Mathematics Subject Classification Numbers. 26D10, 34D40.
\end{abstract}

\section{Introduction}

Integral inequalities which provide explicit bounds on unknown functions play a fundamental role in the development of the theory of differential and integral equations. In the past few years, a number of integral inequalities have been established by many scholars, who are motivated by certain applications such as existence, uniqueness, continuous dependence, comparison, perturbation, boundedness, and stability of solutions of differential and integral equations (see for example [3] and the references cited therein). Among these integral inequalities, we cite the famous Gronwall inequality and its different generalizations ([1], [4], [7], [8], [10], [12]).

In the last few decades, great attention has been paid to automatic control systems and their applications to computational mathematics and modeling. Many problems in control theory correspond to the maximal deviation of the regulated quantity ([11]). Such kinds of problems can be adequately modeled by differential equations that contain the maxima operator. Equations involving maxima of the unknown function are called differential equations with "maxima" ([2], [6]). A. D. Mishkis also points out the necessity of studying differential equations with "maxima" in his survey [9].

The purpose of this paper is to establish some new integral inequalities in the case when maxima of the unknown scalar function is involved in the 
integral. Such inequalities are mathematical tools in the theory of differential equations with "maxima".

\section{Main results}

Let $t_{0} \geq 0$ and $T \geq t_{0}$ be fixed points. Note that $T$ could be equal to $\infty$.

Theorem 1. Let the following conditions be satisfied:

1. The function $\alpha \in C^{1}\left(\left[t_{0}, T\right), \boldsymbol{R}_{+}\right)$is nondecreasing and $\alpha(t) \leq t$.

2. The functions $p, q \in C\left(\left[t_{0}, T\right), \boldsymbol{R}_{+}\right)$and $a, b \in C\left(\left[\alpha\left(t_{0}\right), T\right), \boldsymbol{R}_{+}\right)$.

3. The function $\phi \in C\left(\left[\alpha\left(t_{0}\right)-h, t_{0}\right], \boldsymbol{R}_{+}\right)$.

4. The function $u \in C\left(\left[\alpha\left(t_{0}\right)-h, T\right), \boldsymbol{R}_{+}\right)$satisfies the inequalities

$$
\begin{aligned}
u(t) \leq & k+\int_{t_{0}}^{t}\left[p(s) u(s)+q(s) \max _{\xi \in[s-h, s]} u(\xi)\right] d s \\
& +\int_{\alpha \cdot\left(t_{0}\right)}^{\alpha(t)}\left[a(s) u(s)+b(s) \max _{\xi \in[s-h, s]} u(\xi)\right] d s \quad \text { for } t \in\left[t_{0}, T\right),
\end{aligned}
$$

$$
u(t) \leq \phi(t) \quad \text { for } t \in\left[\alpha\left(t_{0}\right)-h, t_{0}\right],
$$

where $h=$ const $\geq 0, k=$ const $\geq 0$ such that $k \leq \max _{s \in\left[\alpha\left(t_{0}\right)-h, t_{0}\right]} \phi(s)$. Then for $t \in\left[t_{0}, T\right)$, the inequality

$$
u(t) \leq C \exp \left(\int_{t_{0}}^{t}[p(s)+q(s)] d s+\int_{\alpha\left(t_{0}\right)}^{\alpha(t)}[a(s)+b(s)] d s\right)
$$

holds.

Proof. Define a function $v:\left[\alpha\left(t_{0}\right)-h, T\right) \rightarrow \boldsymbol{R}_{+}$by the equality

$$
v(t)=\left\{\begin{aligned}
C+\int_{t_{0}}^{t}\left[p(s) u(s)+q(s) \max _{\xi \in[s-h, s]} u(\xi)\right] d s & \\
\quad+\int_{\alpha\left(t_{0}\right)}^{\alpha(t)}\left[a(s) u(s)+b(s) \max _{\xi \in[s-h, s]} u(\xi)\right] d s, & t \in\left[t_{0}, T\right), \\
C, & t \in\left[\alpha\left(t_{0}\right)-h, t_{0}\right],
\end{aligned}\right.
$$

where $C=\max _{s \in\left[\alpha\left(t_{0}\right)-h, t_{0}\right]} \phi(s)$.

Note that the function $v(t)$ is nondecreasing, $u(t) \leq v(t)$ for $t \in\left[\alpha\left(t_{0}\right)-h, T\right)$ and $\max _{s \in[t-h, t]} v(s)=v(t)$ for $t \in\left[\alpha\left(t_{0}\right), T\right)$. Then from inequality (1) we get for $t \in\left[t_{0}, T\right)$

$$
\begin{aligned}
v(t) \leq & C+\int_{t_{0}}^{t}\left[p(s) v(s)+q(s) \max _{\xi \in[s-h, s]} v(\xi)\right] d s \\
& +\int_{\alpha\left(t_{0}\right)}^{\alpha(t)}\left[a(s) v(s)+b(s) \max _{\xi \in[s-h, s]} v(\xi)\right] d s \\
= & C+\int_{t_{0}}^{t}[p(s)+q(s)] v(s) d s+\int_{\alpha\left(t_{0}\right)}^{\alpha(t)}[a(s)+b(s)] v(s) d s .
\end{aligned}
$$


Substituting the variable $s=\alpha(\eta)$ in the second integral of inequality (4), we use condition 1 for the function $\alpha(t)$ and obtain

$$
\begin{aligned}
v(t) \leq & C+\int_{t_{0}}^{t}[p(s)+q(s)] v(s) d s \\
& +\int_{t_{0}}^{t}[a(\alpha(\eta))+b(\alpha(\eta))] v(\alpha(\eta)) \alpha^{\prime}(\eta) d \eta \\
\leq & C+\int_{t_{0}}^{t}\left[p(s)+q(s)+a(\alpha(s)) \alpha^{\prime}(s)+b(\alpha(s)) \alpha^{\prime}(s)\right] v(s) d s .
\end{aligned}
$$

We apply the Gronwall inequality to (5) and obtain

$$
\begin{aligned}
v(t) & \leq C \exp \left(\int_{t_{0}}^{t}\left[p(s)+q(s)+a(\alpha(s)) \alpha^{\prime}(s)+b(\alpha(s)) \alpha^{\prime}(s)\right] d s\right) \\
& =C \exp \left(\int_{t_{0}}^{t}[p(s)+q(s)] d s+\int_{\alpha\left(t_{0}\right)}^{\alpha(t)}[a(s)+b(s)] d s\right) .
\end{aligned}
$$

Inequality (6) with $u(t) \leq v(t)$ implies the validity of (3).

As a partial case, we obtain the following result for the integral inequality with "maxima":

Corollary 1. Let the following conditions be fulfilled:

1. The functions $p, q \in C\left(\left[t_{0}, T\right), \boldsymbol{R}_{+}\right)$.

2. The function $\phi \in C\left(\left[t_{0}-h, t_{0}\right], \boldsymbol{R}_{+}\right)$.

3. The function $u \in C\left(\left[t_{0}-h, T\right), \boldsymbol{R}_{+}\right)$satisfies the inequalities

$$
\begin{aligned}
& u(t) \leq k+\int_{t_{0}}^{t}\left[p(s) u(s)+q(s) \max _{\xi \in[s-h, s]} u(\xi)\right] d s \quad \text { for } t \in\left[t_{0}, T\right), \\
& u(t) \leq \phi(t) \quad \text { for } t \in\left[t_{0}-h, t_{0}\right],
\end{aligned}
$$

where $h=$ const $\geq 0, k=$ const $\geq 0$ such that $k \leq \max _{s \in\left[t_{0}-h, t_{0}\right]} \phi(s)$. Then for $t \in\left[t_{0}, T\right)$, the inequality

$$
u(t) \leq\left(\max _{s \in\left[t_{0}-h, t_{0}\right]} \phi(s)\right) \exp \left(\int_{t_{0}}^{t}[p(s)+q(s)] d s\right)
$$

holds.

Remark 1. As a partial case of Theorem 1 we obtain a result for integral inequalities without maxima ([10], Theorem 1).

In the case when an increasing function is involved in the right part of inequality (1) instead of a constant we obtain the following upper bound for $u(t)$. 
Theorem 2. Let the following conditions be satisfied:

1. The function $\alpha \in C^{1}\left(\left[t_{0}, T\right), \boldsymbol{R}_{+}\right)$is nondecreasing and $\alpha(t) \leq t$.

2. The functions $p, q \in C\left(\left[t_{0}, T\right), \boldsymbol{R}_{+}\right)$and $a, b \in C\left(\left[\alpha\left(t_{0}\right), T\right), \boldsymbol{R}_{+}\right)$.

3. The function $\phi \in C\left(\left[\alpha\left(t_{0}\right)-h, t_{0}\right], \boldsymbol{R}_{+}\right)$.

4. The function $k \in C\left(\left[t_{0}, T\right),(0, \infty)\right)$ is nondecreasing and the inequality $M=\max _{s \in\left[\alpha\left(t_{0}\right)-h, t_{0}\right]} \phi(s) \leq k\left(t_{0}\right)$ holds.

5. The function $u \in C\left(\left[\alpha\left(t_{0}\right)-h, T\right), \boldsymbol{R}_{+}\right)$satisfies the inequalities

$$
\begin{aligned}
u(t) \leq & k(t)+\int_{t_{0}}^{t}\left[p(s) u(s)+q(s) \max _{\xi \in[s-h, s]} u(\xi)\right] d s \\
& +\int_{\alpha\left(t_{0}\right)}^{\alpha(t)}\left[a(s) u(s)+b(s) \max _{\xi \in[s-h, s]} u(\xi)\right] d s \quad \text { for } t \in\left[t_{0}, T\right),
\end{aligned}
$$

$$
u(t) \leq \phi(t) \quad \text { for } t \in\left[\alpha\left(t_{0}\right)-h, t_{0}\right]
$$

where $h=$ const $\geq 0$. Then for $t \in\left[t_{0}, T\right)$, the inequality

$$
u(t) \leq M \frac{k(t)}{k\left(t_{0}\right)} \exp \left(\int_{t_{0}}^{t}[p(s)+q(s)] d s+\int_{\alpha\left(t_{0}\right)}^{\alpha(t)}[a(s)+b(s)] d s\right)
$$

holds.

Proof. From inequality (7) we obtain for $t \in\left[t_{0}, T\right)$ the inequality

$$
\begin{aligned}
\frac{u(t)}{k(t)} \leq & 1+\int_{t_{0}}^{t}\left[p(s) \frac{u(s)}{k(t)}+q(s) \frac{\max _{\xi \in[s-h, s]} u(\xi)}{k(t)}\right] d s \\
& +\int_{\alpha\left(t_{0}\right)}^{\alpha(t)}\left[a(s) \frac{u(s)}{k(t)}+b(s) \frac{\max _{\xi \in[s-h, s]} u(\xi)}{k(t)}\right] d s .
\end{aligned}
$$

From monotonicity of $k(t)$ we obtain for $t \in\left[t_{0}, T\right)$ and $s \in\left[\alpha\left(t_{0}\right), t\right]$ the inequality

$$
\frac{\max _{\xi \in[s-h, s]} u(\xi)}{k(t)} \leq \frac{\max _{\xi \in[s-h, s]} u(\xi)}{\tilde{k}(s)}=\max _{\xi \in[s-h, s]} \frac{u(\xi)}{\tilde{k}(s)} \leq \max _{\xi \in[s-h, s]} \frac{u(\xi)}{\tilde{k}(\xi)},
$$

where the continuous nondecreasing function $\tilde{k}:\left[\alpha\left(t_{0}\right)-h, T\right) \rightarrow \boldsymbol{R}_{+}$is defined by

$$
\tilde{k}(t)= \begin{cases}k(t) & \text { for } t \in\left[t_{0}, T\right) \\ k\left(t_{0}\right) & \text { for } t \in\left[\alpha\left(t_{0}\right)-h, t_{0}\right] .\end{cases}
$$

Define the function $\varphi:\left[\alpha\left(t_{0}\right)-h, T\right) \rightarrow \boldsymbol{R}_{+}$by the equality $\varphi(t)=u(t) / \tilde{k}(t)$. 
From inequalities (10), (11) and the definition of the function $\varphi(t)$ it follows that

$$
\begin{aligned}
\varphi(t) \leq & 1+\int_{t_{0}}^{t}\left[p(s) \varphi(s)+q(s) \max _{\xi \in[s-h, s]} \varphi(\xi)\right] d s \\
& +\int_{\alpha\left(t_{0}\right)}^{\alpha(t)}\left[a(s) \varphi(s)+b(s) \max _{\xi \in[s-h, s]} \varphi(\xi)\right] d s \quad \text { for } t \in\left[t_{0}, T\right), \\
\varphi(t)= & \frac{u(t)}{k\left(t_{0}\right)} \leq \frac{\phi(t)}{k\left(t_{0}\right)} \quad \text { for } t \in\left[\alpha\left(t_{0}\right)-h, t_{0}\right] .
\end{aligned}
$$

From the definition of the function $\tilde{k}(t)$, and inequalities (12), (13), Theorem 1 implies that for $t \in\left[t_{0}, T\right)$ the inequality

$$
\varphi(t) \leq \frac{M}{k\left(t_{0}\right)} \exp \left(\int_{t_{0}}^{t}[p(s)+q(s)] d s+\int_{\alpha\left(t_{0}\right)}^{\alpha(t)}[a(s)+b(s)] d s\right)
$$

holds.

From inequality (14) and the definition of the functions $\tilde{k}(t)$ and $\varphi(t)$, we obtain inequality (9).

Corollary 2. Let all conditions of Theorem 2 be satisfied, and suppose that the equality $k\left(t_{0}\right)=\max _{s \in\left[\alpha\left(t_{0}\right)-h, t_{0}\right]} \phi(s)=\phi\left(t_{0}\right)$ holds. Then for $t \in\left[t_{0}, T\right)$ the inequality

$$
u(t) \leq k(t) \exp \left(\int_{t_{0}}^{t}[p(s)+q(s)] d s+\int_{\alpha\left(t_{0}\right)}^{\alpha(t)}[a(s)+b(s)] d s\right)
$$

holds.

In the case when the function involved in the right part of inequality (1) is not monotonic, we obtain the following result:

Theorem 3. Let the following conditions be satisfied:

1. The function $\alpha \in C^{1}\left(\left[t_{0}, T\right), \boldsymbol{R}_{+}\right)$is nondecreasing and $\alpha(t) \leq t$.

2. The functions $p, q \in C\left(\left[t_{0}, T\right), \boldsymbol{R}_{+}\right)$and $a, b \in C\left(\left[\alpha\left(t_{0}\right), T\right), \boldsymbol{R}_{+}\right)$.

3. The function $\phi \in C\left(\left[\alpha\left(t_{0}\right)-h, T\right), \boldsymbol{R}_{+}\right), \max _{s \in\left[\alpha\left(t_{0}\right)-h, t_{0}\right]} \phi(s)>0$.

4. The function $u \in C\left(\left[\alpha\left(t_{0}\right)-h, T\right), \boldsymbol{R}_{+}\right)$satisfies the inequalities

$$
\begin{aligned}
u(t) \leq & \phi(t)+\int_{t_{0}}^{t}\left[p(s) u(s)+q(s) \max _{\xi \in[s-h, s]} u(\xi)\right] d s \\
& +\int_{\alpha\left(t_{0}\right)}^{\alpha(t)}\left[a(s) u(s)+b(s) \max _{\xi \in[s-h, s]} u(\xi)\right] d s \quad \text { for } t \in\left[t_{0}, T\right),
\end{aligned}
$$

$$
u(t) \leq \phi(t) \quad \text { for } t \in\left[\alpha\left(t_{0}\right)-h, t_{0}\right]
$$


where $h=$ const $\geq 0$. Then for $t \in\left[t_{0}, T\right)$, the inequality

$$
u(t) \leq \phi(t)+e(t) \exp \left(\int_{t_{0}}^{t}[p(s)+q(s)] d s+\int_{\alpha\left(t_{0}\right)}^{\alpha(t)}[a(s)+b(s)] d s\right)
$$

holds, where $e:\left[t_{0}, T\right) \rightarrow \boldsymbol{R}_{+}$is defined by

$$
\begin{aligned}
e(t)= & \max _{s \in\left[\alpha\left(t_{0}\right)-h, t_{0}\right]} \phi(s)+\int_{t_{0}}^{t}\left[p(s) \phi(s)+q(s) \max _{\xi \in[s-h, s]} \phi(\xi)\right] d s \\
& +\int_{t_{0}}^{\max \left(\alpha(t), t_{0}\right)}\left(a(s) \phi(s)+b(s) \max _{\xi \in[s-h, s]} \phi(\xi)\right) d s .
\end{aligned}
$$

Proof. Define the function $z(t):\left[\alpha\left(t_{0}\right)-h, T\right) \rightarrow \boldsymbol{R}_{+}$by

(20) $z(t)= \begin{cases}\int_{t_{0}}^{t}\left[p(s) u(s)+q(s) \max _{\xi \in[s-h, s]} u(\xi)\right] d s & \\ \quad+\int_{\alpha\left(t_{0}\right)}^{\alpha(t)}\left[a(s) u(s)+b(s) \max _{\xi \in[s-h, s]} u(\xi)\right] d s, & t \in\left[t_{0}, T\right), \\ 0, & t \in\left[\alpha\left(t_{0}\right)-h, t_{0}\right) .\end{cases}$

From inequality (16) and the definition of the function $z(t)$ we have for $t \in\left[\alpha\left(t_{0}\right)-h, T\right)$ that

$$
u(t) \leq \phi(t)+z(t)
$$

Suppose that $t \in\left[t_{0}, T\right)$ satisfies $\alpha(t) \geq t_{0}$. Then from inequality (21) it follows that

$$
\begin{aligned}
\int_{\alpha\left(t_{0}\right)}^{\alpha(t)}\left[a(s) u(s)+b(s) \max _{\xi \in[s-h, s]} u(\xi)\right] d s \\
\leq \int_{\alpha\left(t_{0}\right)}^{t_{0}}\left[a(s) z(s)+b(s) \max _{\xi \in[s-h, s]} z(\xi)\right] d s \\
\quad+\int_{t_{0}}^{\alpha(t)}\left[a(s)(\phi(s)+z(s))+b(s)\left(\max _{\xi \in[s-h, s]} \phi(\xi)+\max _{\xi \in[s-h, s]} z(\xi)\right)\right] d s \\
=\int_{t_{0}}^{\max \left(\alpha(t), t_{0}\right)}\left(a(s) \phi(s)+b(s) \max _{\xi \in[s-h, s]} \phi(\xi)\right) d s \\
\quad+\int_{\alpha\left(t_{0}\right)}^{\alpha(t)}\left(a(s) z(s)+b(s) \max _{\xi \in[s-h, s]} z(\xi)\right) d s .
\end{aligned}
$$


Let $t \in\left[t_{0}, T\right): \alpha(t)<t_{0}$. Then from the definition of the function $z(t)$ we get

$$
\begin{aligned}
\int_{\alpha\left(t_{0}\right)}^{\alpha(t)}\left[a(s) u(s)+b(s) \max _{\xi \in[s-h, s]} u(\xi)\right] d s \\
=\int_{\alpha\left(t_{0}\right)}^{\alpha(t)}\left(a(s) z(s)+b(s) \max _{\xi \in[s-h, s]} z(\xi)\right) d s \\
=\int_{t_{0}}^{\max \left(\alpha(t), t_{0}\right)}\left(a(s) \phi(s)+b(s) \max _{\xi \in[s-h, s]} \phi(\xi)\right) d s \\
\quad+\int_{\alpha\left(t_{0}\right)}^{\alpha(t)}\left(a(s) z(s)+b(s) \max _{\xi \in[s-h, s]} z(\xi)\right) d s .
\end{aligned}
$$

From the definition of the function $z(t)$ and inequalities (22), (23) it follows that

$$
\begin{aligned}
z(t) \leq & e(t)+\int_{t_{0}}^{t}\left[p(s) z(s)+q(s) \max _{\xi \in[s-h, s]} z(\xi)\right] d s \\
& +\int_{\alpha\left(t_{0}\right)}^{\alpha(t)}\left[a(s) z(s)+b(s) \max _{\xi \in[s-h, s]} z(\xi)\right] d s, \quad t \in\left[t_{0}, T\right),
\end{aligned}
$$

$$
z(t) \leq \phi(t), \quad t \in\left[\alpha\left(t_{0}\right)-h, t_{0}\right],
$$

where the function $e(t)$ is defined by equality (19). Note that the function $e(t)$ : $\left[t_{0}, T\right) \rightarrow(0, \infty)$ is nondecreasing for $t \in\left[t_{0}, T\right)$ and $e\left(t_{0}\right)=\max _{s \in\left[\alpha\left(t_{0}\right)-h, t_{0}\right]} \phi(s)$.

From inequalities (24), (25), by Theorem 2 we get

$$
z(t) \leq e(t) \exp \left(\int_{t_{0}}^{t}[p(s)+q(s)] d s+\int_{\alpha\left(t_{0}\right)}^{\alpha(t)}[a(s)+b(s)] d s\right) .
$$

From inequalities (21) and (26) we obtain inequality (18).

Remark 2. Note that in the case when the function $\phi(t)$ is nondecreasing, inequality (15) gives a better upper bound than (18) for the function $u(t)$, since the inequality $e^{x} \leq 1+x e^{x}, x \geq 0$ holds.

Now we will consider an inequality in which the unknown function in the left part is presented in a power.

Theorem 4. Let the following conditions be fulfilled:

1. The conditions 1, 2, 3 of Theorem 2 are satisfied.

2. The function $k \in C\left(\left[t_{0}, T\right),(0, \infty)\right)$ is nondecreasing and the inequality $M=\max _{s \in\left[\alpha\left(t_{0}\right)-h, t_{0}\right]} \phi(s) \leq \sqrt[n]{k\left(t_{0}\right)}$ holds. 
3. The function $u \in C\left(\left[\alpha\left(t_{0}\right)-h, T\right), \boldsymbol{R}_{+}\right)$satisfies the inequalities

$$
\begin{aligned}
u^{n}(t) \leq & k(t)+\int_{t_{0}}^{t}\left[p(s) u(s)+q(s) \max _{\xi \in[s-h, s]} u(\xi)\right] d s \\
& +\int_{\alpha\left(t_{0}\right)}^{\alpha(t)}\left[a(s) u(s)+b(s) \max _{\xi \in[s-h, s]} u(\xi)\right] d s \quad \text { for } t \in\left[t_{0}, T\right),
\end{aligned}
$$

$$
u(t) \leq \phi(t) \quad \text { for } t \in\left[\alpha\left(t_{0}\right)-h, t_{0}\right],
$$

where $h=$ const $\geq 0, n=$ const $>1$. Then for $t \in\left[t_{0}, T\right)$ the inequality

$$
u(t) \leq \sqrt[n]{k(t)}+\left(M+\frac{e(t)}{n\left(k\left(t_{0}\right)\right)^{1-1 / n}}\right) e^{A(t)+B(t)}
$$

holds, where

$$
\begin{gathered}
e(t)=\int_{t_{0}}^{t}\left(p(s) \omega(s)+q(s) \max _{\xi \in[s-h, s]} \omega(\xi)\right) d s \\
+\int_{t_{0}}^{\max \left(\alpha(t), t_{0}\right)}\left(a(s) \omega(s)+b(s) \max _{\xi \in[s-h, s]} \omega(\xi)\right) d s, \\
A(t)=\frac{1}{n} \int_{t_{0}}^{t}(k(s))^{(1-n) / n}[p(s)+q(s)] d s, \\
B(t)=\frac{1}{n} \int_{\alpha\left(t_{0}\right)}^{\alpha(t)}(K(s))^{(1-n) / n}[a(s)+b(s)] d s, \\
K(t)=\left\{\begin{array}{ll}
k(t), & t \in\left[t_{0}, T\right) \\
k\left(t_{0}\right), & t \in\left[\alpha\left(t_{0}\right), t_{0}\right),
\end{array} \quad \begin{array}{ll}
\sqrt[n]{k(t)}, & t \in\left(t_{0}, T\right) \\
M, & t \in\left[t_{0}-h, t_{0}\right] .
\end{array}\right.
\end{gathered}
$$

Proof. Define the function $z(t):\left[\alpha\left(t_{0}\right)-h, T\right) \rightarrow \boldsymbol{R}_{+}$by the equality

$$
z(t)=\left\{\begin{array}{cl}
\frac{\sqrt[n]{k(t)}}{n k(t)}\left(\int_{t_{0}}^{t}\left(p(s) u(s)+q(s) \max _{\xi \in[s-h, s]} u(\xi)\right) d s\right. & \\
\left.\quad+\int_{\alpha\left(t_{0}\right)}^{\alpha(t)}\left(a(s) u(s)+b(s) \max _{\xi \in[s-h, s]} u(\xi)\right) d s\right), & t \in\left[t_{0}, T\right) \\
0, & t \in\left[\alpha\left(t_{0}\right)-h, t_{0}\right) .
\end{array}\right.
$$

From inequality (27) we have for $t \in\left[t_{0}, T\right)$

$$
u^{n}(t) \leq k(t)\left(1+n \frac{z(t)}{\sqrt[n]{k(t)}}\right)
$$

or

$$
u(t) \leq \sqrt[n]{k(t)}\left(1+n \frac{z(t)}{\sqrt[n]{k(t)}}\right)^{1 / n}
$$


Applying Bernoulli's inequality $(1+x)^{a} \leq 1+a x$, where $0<a<1$ and $-1<x$, we observe that

$$
u(t) \leq \sqrt[n]{k(t)}\left(1+\frac{z(t)}{\sqrt[n]{k(t)}}\right)=\sqrt[n]{k(t)}+z(t)=\omega(t)+z(t), \quad t \in\left[t_{0}, T\right)
$$

and

$$
u(t) \leq \phi(t) \leq \phi(t)+z(t)=\omega(t)+z(t), \quad t \in\left[\alpha\left(t_{0}\right)-h, t_{0}\right] .
$$

Therefore,

$$
\max _{\xi \in[s-h, s]} u(\xi) \leq \max _{\xi \in[s-h, s]} \omega(\xi)+\max _{\xi \in[s-h, s]} z(\xi), \quad s \in\left[\alpha\left(t_{0}\right), T\right) .
$$

Suppose that $t \in\left[t_{0}, T\right)$ satisfies $\alpha(t) \geq t_{0}$. Then from inequalities (34), (36) we get

$$
\begin{aligned}
\int_{\alpha\left(t_{0}\right)}^{\alpha(t)}\left[a(s) u(s)+b(s) \max _{\xi \in[s-h, s]} u(\xi)\right] d s & \\
\leq & \int_{\alpha\left(t_{0}\right)}^{t_{0}}\left[a(s) z(s)+b(s) \max _{\xi \in[s-h, s]} z(\xi)\right] d s \\
& +\int_{t_{0}}^{\alpha(t)}\left[a(s)(\omega(s)+z(s))+b(s)\left(\max _{\xi \in[s-h, s]} \omega(\xi)+\max _{\xi \in[s-h, s]} z(\xi)\right)\right] d s \\
= & \int_{t_{0}}^{\max \left(\alpha(t), t_{0}\right)}\left(a(s) \omega(s)+b(s) \max _{\xi \in[s-h, s]} \omega(\xi)\right) d s \\
& +\int_{\alpha\left(t_{0}\right)}^{\alpha(t)}\left(a(s) z(s)+b(s) \max _{\xi \in[s-h, s]} z(\xi)\right) d s .
\end{aligned}
$$

Suppose that $t \in\left[t_{0}, T\right)$ satisfies $\alpha(t)<t_{0}$. Then from the definition of the function $z(t)$ and inequalities (34), (36) we get

$$
\begin{aligned}
\int_{\alpha\left(t_{0}\right)}^{\alpha(t)}\left[a(s) u(s)+b(s) \max _{\xi \in[s-h, s]} u(\xi)\right] d s \\
=\int_{\alpha\left(t_{0}\right)}^{\alpha(t)}\left(a(s) z(s)+b(s) \max _{\xi \in[s-h, s]} z(\xi)\right) d s \\
=\int_{t_{0}}^{\max \left(\alpha(t), t_{0}\right)}\left(a(s) \omega(s)+b(s) \max _{\xi \in[s-h, s]} \omega(\xi)\right) d s \\
+\int_{\alpha \cdot\left(t_{0}\right)}^{\alpha(t)}\left(a(s) z(s)+b(s) \max _{\xi \in[s-h, s]} z(\xi)\right) d s .
\end{aligned}
$$


Let $C=\operatorname{Mnk}\left(t_{0}\right)^{1-1 / n}>0$. Note that the function $v:\left[t_{0}, T\right) \rightarrow(0, \infty)$, $v(t)=\left(n k\left(t_{0}\right)^{1-1 / n}\right)^{-1}(C+e(t))$ is nondecreasing and the equality $v\left(t_{0}\right)=$ $\left(n k\left(t_{0}\right)^{1-1 / n}\right)^{-1}\left(C+e\left(t_{0}\right)\right)=M$ holds, where the function $e(t)$ is defined by $(30)$. From the definition of the function $z(t)$ and inequalities (37), (38), it follows that

$$
\begin{aligned}
z(t) \leq & \frac{1}{n k(t)^{1-1 / n}}\left(e(t)+\int_{t_{0}}^{t}\left[p(s) z(s)+q(s) \max _{\xi \in[s-h, s]} z(\xi)\right] d s\right. \\
& \left.\quad+\int_{\alpha\left(t_{0}\right)}^{\alpha(t)}\left[a(s) z(s)+b(s) \max _{\xi \in[s-h, s]} z(\xi)\right] d s\right) \\
\leq & \frac{1}{n k\left(t_{0}\right)^{1-1 / n}}(C+e(t)) \\
& +\frac{1}{n k(t)^{1-1 / n}} \int_{t_{0}}^{t}\left[p(s) z(s)+q(s) \max _{\xi \in[s-h, s]} z(\xi)\right] d s \\
& +\frac{1}{n k(t)^{1-1 / n}} \int_{\alpha\left(t_{0}\right)}^{\alpha(t)}\left[a(s) z(s)+b(s) \max _{\xi \in[s-h, s]} z(\xi)\right] d s \\
\leq & v(t)+\int_{t_{0}}^{t} \frac{1}{n}\left[\frac{p(s)}{(k(s))^{1-1 / n}} z(s)+\frac{q(s)}{(k(s))^{1-1 / n}} \max _{\xi \in[s-h, s]} z(\xi)\right] d s \\
& +\int_{\alpha\left(t_{0}\right)}^{\alpha(t)} \frac{1}{n}\left[\frac{a(s)}{(K(s))^{1-1 / n}} z(s)+\frac{b(s)}{(K(s))^{1-1 / n}} \max _{\xi \in[s-h, s]} z(\xi)\right] d s, \quad t \in\left[t_{0}, T\right),
\end{aligned}
$$

(40) $z(t) \leq \phi(t), \quad t \in\left[\alpha\left(t_{0}\right)-h, t_{0}\right]$.

From inequalities (39), (40), Theorem 2 implies

$$
z(t) \leq M \frac{v(t)}{v\left(t_{0}\right)} e^{A(t)+B(t)}=\left(M+\frac{e(t)}{n\left(k\left(t_{0}\right)\right)^{1-1 / n}}\right) e^{A(t)+B(t)},
$$

where $A(t)$ and $B(t)$ are defined by (31) and (32), respectively.

Substituting the bound (41) for $z(t)$ into the right part of (34), we obtain inequality (29).

Remark 3. As partial cases of Theorem 3 and Theorem 4 we obtain results for integral inequalities without maxima ([8], Theorem 2.1 and Theorem 2.2).

\section{Applications}

We will apply some of the above inequalities to study properties of solutions of differential equations with "maxima". Note that differential equations 
with "maxima" are adequate models of real processes whose present state depends on the maximum of its deviation in the past. Such kinds of problems are, for example, Hausrath equation [13], the model describing the vision process in the compound eye ([5]), and the model of a generator ([11]). On the other hand, it is relevant to mention here the opinion of Myshkis that "the specific character of these equations is not yet sufficiently clear". In his survey [9] he also distinguishes the equations with maxima as differential equations with deviating argument of complex structure.

Example 1. Consider the following system of differential equations with a "maximum"

$$
x^{\prime}=f\left(t, x(t), \max _{s \in[\beta(t), \alpha(t)]} x(s)\right) \quad \text { for } t \geq t_{0}
$$

with initial condition

$$
x(t)=\varphi(t) \quad \text { for } t \in\left[\alpha\left(t_{0}\right)-h, t_{0}\right],
$$

where $x \in \boldsymbol{R}^{n}, \varphi:\left[\alpha\left(t_{0}\right)-h, t_{0}\right] \rightarrow \boldsymbol{R}^{n}, f:[0, \infty) \times \boldsymbol{R}^{n} \times \boldsymbol{R}^{n} \rightarrow \boldsymbol{R}^{n}, h>0$ is a constant.

Theorem 5 (Uniqueness). Let the following conditions be satisfied:

1. The functions $\alpha, \beta \in C\left(\left[t_{0}, \infty\right), \boldsymbol{R}_{+}\right), \alpha(t)$ is an increasing function, $\beta(t) \leq \alpha(t) \leq t$, and there exists a constant $h>0$ such that $0<\alpha(t)-\beta(t) \leq h$ for $t \geq t_{0}$.

2. The function $f \in C\left(\left[t_{0}, \infty\right) \times \boldsymbol{R}^{n} \times \boldsymbol{R}^{n}, \boldsymbol{R}^{n}\right)$ satisfies the condition

$$
\left\|f\left(t, x_{1}, y_{1}\right)-f\left(t, x_{2}, y_{2}\right)\right\| \leq g(t)\left\|x_{1}-x_{2}\right\|+r(t)\left\|y_{1}-y_{2}\right\|,
$$

for $t \geq t_{0}, x_{i}, y_{i} \in \boldsymbol{R}^{n}(i=1,2)$, where $g(t), r(t) \in C\left(\left[t_{0}, \infty\right), \boldsymbol{R}_{+}\right)$.

3. For any initial function $\varphi \in C\left(\left[\alpha\left(t_{0}\right)-h, t_{0}\right], R^{n}\right)$, the initial value problem (42), (43) has at least one solution $x\left(t ; t_{0}, \varphi\right)$ defined for $t \geq \alpha\left(t_{0}\right)-h$.

Then initial value problem (42), (43) has exactly one solution.

Proof. Let $\varphi \in C\left(\left[\alpha\left(t_{0}\right)-h, t_{0}\right], \boldsymbol{R}^{n}\right)$ be a fixed initial function. Assume there exist two different solutions $u(t)=u\left(t ; t_{0}, \varphi\right)$ and $v(t)=v\left(t ; t_{0}, \varphi\right)$ of the initial value problem (42), (43), that are defined for $t \geq \alpha\left(t_{0}\right)-h$. Both functions $u(t)$ and $v(t)$ satisfy the integral equations

$$
\begin{array}{ll}
u(t)=\varphi\left(t_{0}\right)+\int_{t_{0}}^{t} f\left(s, u(s), \max _{\xi \in[\beta(s), \alpha(s)]} u(\xi)\right) d s & \text { for } t \geq t_{0}, \\
v(t)=\varphi\left(t_{0}\right)+\int_{t_{0}}^{t} f\left(s, v(s), \max _{\xi \in[\beta(s), \alpha(s)]} v(\xi)\right) d s \quad \text { for } t \geq t_{0}
\end{array}
$$

and $u(t)=v(t)=\varphi(t)$ for $t \in\left[\alpha\left(t_{0}\right)-h, t_{0}\right]$. 
Then the norm of the difference of both solutions $u(t)$ and $v(t)$ satisfies the inequalities

$$
\begin{aligned}
\| u(t) & -v(t) \| \\
& \leq \int_{t_{0}}^{t}\left\|f\left(s, u(s), \max _{\xi \in[\beta(s), \alpha(s)]} u(\xi)\right)-f\left(s, v(s), \max _{\xi \in[\beta(s), \alpha(s)]} v(\xi)\right)\right\| d s \\
& \leq \int_{t_{0}}^{t}\left(g(s)\|u(s)-v(s)\|+r(s)\left\|\max _{\xi \in[\beta(s), \alpha(s)]} u(\xi)-\max _{\xi \in[\beta(s), \alpha(s)]} v(\xi)\right\|\right) d s \\
& \leq \int_{t_{0}}^{t} g(s)\|u(s)-v(s)\| d s+\int_{t_{0}}^{t} r(s) \max _{\xi \in[\beta(s), \alpha(s)]}\|u(\xi)-v(\xi)\| d s, \quad t \geq t_{0},
\end{aligned}
$$

Set $z(t)=\|u(t)-v(t)\| \quad$ for $\quad t \in\left[\alpha\left(t_{0}\right)-h, \infty\right)$ and change the variable $s=\alpha^{-1}(\eta)$ in the second integral of (44). Then use the inequality $\max _{\xi \in[\beta(t), \alpha(t)]} z(\xi) \leq \max _{\xi \in[\alpha(t)-h, \alpha(t)]} z(\xi)$ that follows from condition 1 of Theorem 5 to obtain the inequality

$$
z(t) \leq \int_{t_{0}}^{t} g(s) z(s) d s+\int_{\alpha\left(t_{0}\right)}^{\alpha(t)} r\left(\alpha^{-1}(\eta)\right)\left(\alpha^{-1}(\eta)\right)^{\prime} \max _{\xi \in[\eta-h, \eta]} z(\xi) d \eta, \quad t \geq t_{0} .
$$

From inequality (45) and Theorem 1 for $\phi(t) \equiv 0$ on $\left[\alpha\left(t_{0}\right)-h, t_{0}\right], k=0$, $p(t)=g(t), \quad q(t) \equiv 0 \quad$ on $\left[t_{0}, \infty\right)$ and $a(t) \equiv 0, \quad b(t)=r\left(\alpha^{-1}(t)\right)\left(\alpha^{-1}(t)\right)^{\prime} \quad$ on $\left[\alpha\left(t_{0}\right), \infty\right)$, we obtain $z(t) \leq 0$ for $t \geq t_{0}$, which proves the validity of the equality $\|u(t)-v(t)\|=0$ for $t \geq t_{0}$ or $u(t) \equiv v(t)$.

Example 2. Consider the following scalar differential equation with a "maximum"

$$
x x^{\prime}=f\left(t, x(t), \max _{s \in[\beta(t), \alpha(t)]} x(s)\right) \quad \text { for } t \geq t_{0}
$$

with initial condition

$$
x(t)=\varphi(t) \quad \text { for } t \in\left[\alpha\left(t_{0}\right)-h, t_{0}\right],
$$

where $x \in \boldsymbol{R}, \varphi:\left[\alpha\left(t_{0}\right)-h, t_{0}\right] \rightarrow \boldsymbol{R}, f:[0, \infty) \times \boldsymbol{R} \times \boldsymbol{R} \rightarrow \boldsymbol{R}, h>0$ is a constant.

Theorem 6 (Upper bound). Let the following conditions be satisfied:

1. The functions $\alpha, \beta \in C\left(\left[t_{0}, \infty\right), \boldsymbol{R}_{+}\right), \alpha(t)$ is an increasing function, $\beta(t) \leq \alpha(t) \leq t$ and there exists a constant $h>0$ such that $0<\alpha(t)-\beta(t) \leq h$ for $t \geq t_{0}$.

2. The function $f \in C\left(\left[t_{0}, \infty\right) \times \boldsymbol{R} \times \boldsymbol{R}, \boldsymbol{R}\right), f(t, 0,0) \equiv 0$, satisfies the condition

$$
\left|f\left(t, x_{1}, y_{1}\right)-f\left(t, x_{2}, y_{2}\right)\right| \leq g(t)\left|x_{1}-x_{2}\right|+r(t)\left|y_{1}-y_{2}\right|,
$$

for $t \geq t_{0}, x_{i}, y_{i} \in \boldsymbol{R}(i=1,2)$, where $g(t), r(t) \in C\left(\left[t_{0}, \infty\right), \boldsymbol{R}_{+}\right)$. 
3. The function $\varphi \in C\left(\left[\alpha\left(t_{0}\right)-h, t_{0}\right], \boldsymbol{R}\right)$ satisfies $\left|\varphi\left(t_{0}\right)\right|>0$.

4. The solution $x\left(t ; t_{0}, \varphi\right)$ of the initial value problem (46), (47) is defined for $t \geq \alpha\left(t_{0}\right)-h$.

Then for $t \geq t_{0}$ the inequality

$$
\left|x\left(t ; t_{0}, \varphi\right)\right| \leq\left|\varphi\left(t_{0}\right)\right|+M\left(1+\frac{1}{\left|\varphi\left(t_{0}\right)\right|} \int_{t_{0}}^{t}(g(s)+r(s)) d s\right) e^{\int_{t_{0}}^{t}(g(s)+r(s)) d s /\left|\varphi\left(t_{0}\right)\right|}
$$

holds, where $M=\max _{s \in\left[\alpha\left(t_{0}\right)-h, t_{0}\right]}|\varphi(s)|$.

Proof. The function $x(t)=x\left(t ; t_{0}, \varphi\right)$ satisfies the following integral equation

$$
\begin{aligned}
& (x(t))^{2}=\left(\varphi\left(t_{0}\right)\right)^{2}+\int_{t_{0}}^{t} 2 f\left(s, x(s), \max _{\xi \in[\beta(s), \alpha(s)]} x(\xi)\right) d s \quad \text { for } t \geq t_{0}, \\
& x(t)=\varphi(t), \quad t \in\left[\alpha\left(t_{0}\right)-h, t_{0}\right] .
\end{aligned}
$$

According to condition 2 of Theorem 6 we get

$$
\begin{aligned}
|x(t)|^{2} & \leq\left|\varphi\left(t_{0}\right)\right|^{2}+2 \int_{t_{0}}^{t}\left|f\left(s, x(s), \max _{\xi \in[\beta(s), \alpha(s)]} x(\xi)\right)\right| d s \\
& \leq\left|\varphi\left(t_{0}\right)\right|^{2}+2 \int_{t_{0}}^{t} g(s)|x(s)| d s+2 \int_{t_{0}}^{t} r(s) \max _{\xi \in[\beta(s), \alpha(s)]}|x(\xi)| d s, \quad t \geq t_{0},
\end{aligned}
$$

Setting $u(t)=|x(t)|$ for $t \in\left[\alpha\left(t_{0}\right)-h, \infty\right)$, substituting the variable $s=\alpha^{-1}(\eta)$ into the second integral of (49), and using the inequality $\max _{\xi \in[\beta(t), \alpha(t)]} u(\xi) \leq$ $\max _{\xi \in[\alpha(t)-h, \alpha(t)]} u(\xi)$ that follows from condition 1 of Theorem 6 , we obtain for $t \geq t_{0}$ the following inequality

$$
\begin{aligned}
(u(t))^{2} \leq & \left|\varphi\left(t_{0}\right)\right|^{2}+\int_{t_{0}}^{t} 2 g(s) u(s) d s \\
& +\int_{\alpha\left(t_{0}\right)}^{\alpha(t)} 2 r\left(\alpha^{-1}(\eta)\right)\left(\alpha^{-1}(\eta)\right)^{\prime} \max _{\xi \in[\eta-h, \eta]} u(\xi) d \eta .
\end{aligned}
$$

From inequality (50), by Theorem 4 for $n=2, \phi(t)=|\varphi(t)|$ on $\left[\alpha\left(t_{0}\right)-h, t_{0}\right]$, $k(t)=\left|\varphi\left(t_{0}\right)\right|^{2}>0, \quad p(t)=2 g(t), \quad q(t) \equiv 0 \quad$ on $\left[t_{0}, \infty\right), \quad$ and $a(t) \equiv 0, \quad b(t)=$ $2 r\left(\alpha^{-1}(t)\right)\left(\alpha^{-1}(t)\right)^{\prime}$ on $\left[\alpha\left(t_{0}\right), \infty\right)$, we obtain the inequality

$$
u(t) \leq\left|\varphi\left(t_{0}\right)\right|+\left(M+\frac{e(t)}{2\left|\varphi\left(t_{0}\right)\right|}\right) e^{\int_{t_{0}}^{t}(g(s)+r(s)) d s /\left|\varphi\left(t_{0}\right)\right|},
$$

where

$$
e(t)=2 M \int_{t_{0}}^{t}(g(s)+r(s)) d s .
$$

Inequality (51) proves the validity of inequality (48). 
Acknowledgement. This research was partially supported by RS09FMI018.

\title{
References
}

[1] Agarwal, Ravi P., Deng, S. and Zhang, W., Generalization of a retarded Gronwall-like inequality and its applications, Appl. Math. Comput., 165 (2005), 599-612.

[2] Angelov, V. G. and Bainov, D. D., On the functional differential equations with "maximums", Applicable Anal., 16 (1983), 187-194.

[3] Bainov, D. D. and Simeonov, P. S., Integral inequalities and applications, Kluwer, 1992.

[4] Cho, Y., Kim, Young-Ho and Pecaric, J., New Gronwall-Ou-Iang type integral inequalities and their applications, ANZIAM J., 50 (2008), 111-127.

[5] Hadeler, K. P., Delay equations in biology, Lecture Notes in Math., 730, New York, Springer, 1979, pp. 136-156.

[6] Hristova, S. G. and Roberts, L. F., Boundedness of the solutions of differential equations with "maxima", Int. J. Appl. Math., 4 (2000), 231-240.

[7] Ma, Q. and Pecaric, J., On certain new nonlinear retarded integral inequalities for functions in two variables and their applications, J. Korean Math. Soc., 45 (2008), 121-136.

[ 8 ] Kim, Y. H., On some new integral inequalities for functions in one and two variables, Acta Math. Sin. (Engl. Ser.), 21 (2005), 423-434.

[9] Mishkis, A. D., On some problems of the theory of differential equations with deviating argument, Russian Math. Surveys, 32 (1977), 181-210 (in Russian).

[10] Pachpatte, B. G., Explicit bounds on certain integral inequalities, J. Math. Anal. Appl., 267 (2002), 48-61.

[11] Popov, E. P., Automatic regulation and control, Moscow, 1966 (in Russian).

[12] Zhang, W. and Deng, S., Projected Gronwall-Bellman's inequality for integrable functions, Math. Comput. Modeling, 34 (2001), 393-402.

\author{
nuna adreso: \\ Snezhana G. Hristova \\ Department of Applied Mathematics and \\ Modeling \\ Plovdiv University \\ 4000 Plovdiv \\ Bulgaria \\ E-mail: snehri@uni-plovdiv.bg \\ Kremena V. Stefanova \\ Department of Applied Mathematics and \\ Modeling \\ Plovdiv University \\ 4000 Plovdiv \\ Bulgaria
}

(Ricevita la 7-an de aŭgusto, 2009)

(Reviziita la 23-an de septembro, 2009) 